\title{
Erwerb der L2-Artikulation im Erwachsenenalter: einige offene Fragen
}

\author{
Birgit Gunsenheimer
}

It is a well-known phenomenon that adult learners face greater difficulties in acquiring an accent-free command of a foreign language than children. Some approaches even claim that this is impossible. However, occasional researches within the last decades have shown that there are cases in which late learners achieve a level in pronunciation that is judged as authentic by native speakers. This contribution focuses on the question, how fundamental the restrictedness of adult learners in achieving a native accent of the foreign language really is and specifies some of the factors which ought to receive further consideration in both research and teaching.

articulation - pronunciation - foreign accent - native pronunciation - phonology - second language learning - language acquisition - critical period hypothesis - attitudes

Artikulation-Aussprache-Akzent-akzentfreiesSprechen-Phonologie-Fremdspracherwerb Spracherwerbsforschung - Psycholinguistik - kritische Periode - Attitüden

\section{Einleitung}

Die Diskussion, ob der L2-Erwerb im Erwachsenenalter erschwert ist und warum beim Zweit- oder Fremdspracherwerb ${ }^{1}$ in einer Lebensphase ab der Pubertät meistens ein Akzent bleibt, ist seit dem Formulieren der Critical Period Hypothesis von Lenneberg im Jahr 1967 (LENNEBERG: 1967) nicht abgerissen. Obgleich in der Fachliteratur nicht unbedingter Konsens herrscht, werden in verschiedenen Ansätzen bestimmte Grundaussagen gleichermaßen vertreten. Die generelle Existenz einer kritischen Periode beim Fremdspracherwerb wird nicht mehr uneingeschränkt angenommen. ${ }^{2}$ Unterschiedliche Begriffe und Konzeptionen sind Bestandteil des Diskurses, so wird zum Beispiel von manchen Autoren nicht mehr von einer kritischen Periode, sondern von einer sensitiven Periode oder einem optimalen Alter (so z. B. OSAAR: 2003, 54 f.) gesprochen, was ein flexibleres Konzept und keine Ausschließlichkeit bedeutet. Erwachsene, so die Vertreter zahlreicher aktueller Positionen, können sehr wohl eine Fremdsprache bis zur Perfektion lernen. Es kann noch nicht einmal von erheblich größeren Schwierigkeiten als im Kindesalter gesprochen werden, da

1 Der üblichen Unterscheidung zwischen Zweit- und Fremdspracherwerb in der Fachliteratur schließt sich die Verfasserin im Prinzip an. Aus Gründen der Einfachheit und da sonst immer beide Begriffe parallel benutzt werden müssten, wird in diesem Artikel fortan der Begriff „Fremdsprache“ in umfassenderem Sinn verwendet, d.h. er schließt das Spezifikum der Zweitsprache mit ein.

2 Einen Überblick über den Forschungsstand hinsichtlich der Hypothese von der kritischen Periode gibt OKSAAR (2003, 53 f). Eine ausführliche Diskussion in Form unterschiedlich ausgerichteter Beiträge findet sich im Sammelband von BIRDSONG (1999). 
z. B. die kognitive Entwicklung weiter ist und dadurch Strukturen besser erfasst werden können. Dies betrifft alle Fertigkeiten - mit Ausnahme der Aussprache. Diese spielt, wie von zahlreichen Werken auf den Gebieten der Psycholinguistik und des Fremdspracherwerbs zumindest erwähnt, wenn auch nicht weiter diskutiert wird, eine gesonderte Rolle. Für sie scheint weiterhin zuzutreffen, was zuvor von den Vertretern der Critical Period Hypothesis als allgemeingültig dargestellt wurde: Nach dem Überschreiten einer bestimmten Altersgrenze ist es für die Lerner sehr schwierig bzw. unmöglich, muttersprachliche Kompetenz auf dem Gebiet der Aussprache zu erwerben. Wie Els Oksaar konstatiert, „herrscht in der einschlägigen Forschung Einigkeit darüber, dass der fremde Akzent bei Erwachsenen viel häufiger festzustellen ist als bei Kindern" (OKSAAR: 2003, 64).

Bei der Frage danach, wo genau die Gründe liegen oder wie geeignete Lehr- und Lernmethoden Abhilfe schaffen könnten, erhält man jedoch nur spärlich Antwort. Und da die Alltagserfahrung zwar bestätigt, dass die meisten erwachsenen Lerner einen Akzent behalten, es aber doch immer wieder die berühmten Ausnahmen gibt, stellt sich eine weitere, grundsätzliche Frage: Trifft die Annahme, dass ein akzentfreies Erlernen der L2 im Erwachsenenalter generell nicht möglich ist, überhaupt zu?

Im Jahr 1979 wurde eine Untersuchung von Gerald Neufeld (NEUFELD: 1979) veröffentlicht, die im Zusammenhang mit der soeben skizzierten Problemstellung bis heute gelegentlich in der Fachliteratur zitiert wird, da sie bis Mitte der Neunziger Jahre eine Ausnahmeerscheinung darstellte. Neufeld stellte in einer Versuchsreihe fest, dass erwachsene Lerner in der Lage waren, nach intensiver Hör- und Ausspracheschulung einzelne Äußerungen in einer ihnen unbekannten Sprache so auszusprechen, dass sie von muttersprachlichen Kontrollpersonen als ebenfalls muttersprachlich eingestuft wurden. Dieser Versuch und die entsprechenden Beobachtungen blieb jedoch in der Folgezeit erstens lange ein Einzelfall und zweitens nicht ohne Kritik (s. BONGAERTS et al.: 1997, 450 u. GASS/SELINKER: 2001, 336 f.). Grundsätzlich herrschte lange Zeit die Meinung vor und gilt bis heute, dass erwachsene Lernende, die sich einen von Muttersprachlern nicht zu unterscheidenden Akzent aneignen, eine bemerkenswerte Ausnahme im Zehntelprozentbereich darstellen (s. BONGAERTS et al.: 1997, 448).

Die Frage, ob erwachsene Lerner wirklich grundsätzlich nicht in der Lage sind, eine Fremdsprache akzentfrei zu erlernen, ist meiner Ansicht nach bisher nicht befriedigend beantwortet worden. In nahezu allen in den letzten Jahren erschienenen Werken zur Psycholinguistik und zum Fremdspracherwerb wird stets rezitiert, was oben beschrieben wurde: Im Regelfall ist dieses Ziel nicht zu erreichen, von manchen Lernern aber eben doch. Diese unscharfe Aussage wirft die weitere Frage auf, unter welchen Bedingungen ein erwachsener Lerner das Ziel der authentischen Aussprache erreichen kann. Diese sind in der Forschungsliteratur teilweise bereits benannt worden. 


\section{Beiträge aus der Fachliteratur}

Ein Grund, warum eine umfassende Untersuchung zu den soeben formulierten Fragen fehlt, mag darin liegen, dass dazu verschiedene Nachbar- und Referenzdisziplinen einbezogen werden müssen, die jedoch in sich hochspezialisiert sind und weitgehend für sich arbeiten. Die derzeitigen Errungenschaften in Phonetik, Phonologie, Psycholinguistik und Spracherwerbsforschung, aber auch in der Biologie und cerebralen Hirnforschung schlagen sich in Fachartikeln nieder, die für das Thema relevant wären. Diese sind jedoch terminologisch und methodologisch zum Teil so spezifiziert, dass sie von Vertretern anderer Disziplinen kaum mehr gelesen werden können. Wegen der Flut an wissenschaftlichen Artikeln, die jedes Jahr erscheinen, dürften allein schon Zeitgründe ein Hindernis sein (vgl. dazu auch KLEIN: 2008, 12).

Einen guten Überblick über einzelne Forschungsergebnisse und Positionen bis zur Mitte der Neunziger Jahre geben Bongaerts, van Summeren, Planke und Schils ${ }^{1}$ (in diesem Beitrag stets zitiert als Bongaerts et al.; BONGAERTS et al.: 1997, 448 f.). Interessante Positionen sind zum Beispiel die von Seliger, Walsh und Diller, die „,concurred in suggesting that there is not one critical period affecting all aspects of language at the same time but many critical periods, each closing off different abilities, the ability to master a native accent in a foreign language being the first to be lost, around the onset of puberty" (ebd., 448). Im weiteren Verlauf wird Scovel zitiert, der von einer kritischen Periode lediglich für den Erwerb der Aussprache ausgeht. Beide Positionen wären plausible Erklärungen dafür, warum das Erlernen einer Fremdsprache im Erwachsenenalter gelingt - bis auf die authentische Aussprache.

In diesem Zusammenhang ist auch eine verwandte Fragestellung aus der Psycholinguistik zu nennen, die nach der Gültigkeit zweier unterschiedlicher Modelle des Spracherwerbs, des Plateaumodells und des Permanenzmodells (FIEHLER: 2003, 812). Ersteres geht davon aus, dass der Spracherwerb zu einem Abschluss kommt, letzteres davon, dass lebenslanges Lernen involviert ist. Für den Erstspracherwerb könnten beide Modelle unterschiedliche Aspekte erklären, den Aufbau eines grammatischen Systems einerseits und die ständige Erweiterbarkeit von kommunikativen Fähigkeiten und Wortschatz andererseits. Es ist nun die Frage, ob diese Modelle auch auf den Fremdspracherwerb, in unserem Zusammenhang speziell auf das Modifizieren von Aussprachemustern, anwendbar sind. Da es Fiehler in seinem Beitrag allerdings um Erweiterung der L1 geht, kommt diese Fragestellung bei ihm nicht vor. Jordens, der im selben Sammelband über Constraints beim L2Spracherwerb spricht, geht dagegen klar von einer Fossilierung aus: „Learners are

1 Ähnliche Zusammenfassungen finden sich auch im Sammelband von Birdsong (1999). Dort stellt in einem Kapitel auch Theo Bongaerts seine bereits vorher veröffentlichten Untersuchungen noch einmal vor. 
hardly ever able to reach native competence“. Dies gelte sowohl für den natürlichen Spracherwerb als auch für "classroom situations“ (JORDENS: 2003, 827). Die bereits erwähnten Ausnahmen nennt er „a few anecdotal cases“ (ebd.). Seine Bemerkungen über den Erwerb der fremdsprachigen Phonologie gestaltet er sehr knapp, indem er die Positionen von Henning Wode zusammenfasst (JORDENS: 2003, 829; vgl. WODE: 1996). Der fremdsprachliche Akzent wird nämlich von diesem auf eine Wahrnehmungsweise zurückgeführt, die vom Entwickeln eines phonologischen Systems in der Erstsprache herrührt. Dadurch bildet sich die Fähigkeit, kleine Unterschiede zwischen einzelnen Lauten, die in der L1 nicht phonematisch relevant sind, zurück; sie werden also nicht mehr wahrgenommen, auch wenn sie in der L2 sehr wohl phonologisch ausschlaggebend sind. Insofern kann auch kein entsprechendes System für die zweite Sprache aufgebaut werden, die Laute werden vom phonologischen System der Erstsprache beeinflusst. Jordens kommt zu dem Schluss: „Hence, it is the perceptual difficulties of L2 learners which are the cause of foreign accent in L2 production“" (JORDENS: 2003, 829).

Ansonsten findet man in Werken zur Psycholinguistik sehr wenig über die Besonderheiten des Lernens der Aussprache im Erwachsenenalter. In Butzkamms „Psycholinguistik des Fremdsprachenunterrichts" etwa kommt das Thema Artikulation überhaupt nicht vor.

Erklärungsansätze verschiedenster Art finden sich dagegen in den HSK-Bänden „Deutsch als Fremdsprache“. Was Fossilierung betrifft, so geht Apeltauer vom Konzept einer Lernersprache als individuellen System aus, das sich bei geeignetem Input und mit entsprechender Motivation entwickeln kann (APELTAUER: 2001, 678). Fossilierung nennt er zwar als Auswirkung der Tatsache, dass dieses sprachliche System sehr störanfällig ist. Andererseits räumt er ein, dass das nach ein paar Jahren typischerweise auftretende sog. Lernplateau nicht der unbedingte Endpunkt sein muss. „Manche Lerner überwinden diesen Punkt jedoch und erreichen später in ihrer Fremdsprache eine nahezu muttersprachliche Kompetenz" (ebd.). Unter welchen Umständen dies der Fall ist, wird nicht genannt; vor allem geht Apeltauer nicht speziell auf die Problematik der Aussprache ein.

Im selben Band finden sich jedoch Antworten auf die Frage, warum gerade die Aussprache als die einzige Komponente gilt, die vom Erwachsenen im Regelfall nicht mehr vollständig zu perfektionieren ist und was sie von den Fertigkeiten wie Schreiben, Lesen oder Hören unterscheidet. Sie ist die einzige Komponente, bei denen die Motorik eine entscheidende Rolle spielt (wenn man die Bewegungen beim Schreiben außer Betracht lässt).

Inwieweit ist nämlich das erschwerte Erlernen der Aussprache einer L2 im Erwachsenenalter vergleichbar mit anderen Bewegungsabläufen, also Fertigkeiten, die stark auf Motorik und Muskelkoordination basieren? Mit anderen Worten: Davon ausgehend, dass Aussprache mit Bewegung zu tun hat, entspricht ein solcher Lernprozess vielleicht dem Sich-Aneignen einer ganz neuen Art der Bewegung bzw. 
neuen Bewegungsabfolge, etwa beim Erlernen von Jazztanz oder dem Klavierspielen? Liegen die spezifischen Schwierigkeiten bei der Neukoordination einzelner Muskelgruppen oder des ganzen Körpers vor allem im motorischen Bereich, sind sie eher neurolingual zu sehen, oder sind diese Fragen überhaupt nicht voreinander zu trennen? Ist es möglich, dass nicht solche, sondern psychologische Faktoren eine entscheidende Rolle spielen? Es gibt keine Starpianisten, die im fortgeschrittenen Alter angefangen haben, das Instrument zu lernen. Ist das ein Beweis dafür, dass bestimmte Bewegungsabläufe nicht mehr erworben werden können, jedenfalls nicht in dem Grad, dass sie fließend und schnell genug sind, oder ist die Motivation in früheren Jahren das entscheidende Kriterium? Ist der Lerner fokussierter und verwendet er mehr Energie, weil er das Ziel der Meisterschaft vor Augen hat, und findet das im Alter nicht mehr statt?

In einem Beitrag über biologische und neurophysiologische Grundlagen des L2-Lernens stellt Schönpflug fest: „Die Beantwortung der Frage nach einer kritischen Periode für den Zweitspracherwerb, insbesondere für die Aussprache, sollte nicht getrennt von anderen vergleichbaren perzeptuomotorischen Lernvorgängen betrachtet werden. Wenn es für perzeptuomotorische Fertigkeiten wie z. B. Klavierspielen eine kritische Lernperiode gibt, könnte dies auch für den Zweitspracherwerb wahrscheinlich sein" (SCHÖNPFLUG: 2001, 701). Diese Aussage ist nicht grundlos vorsichtig formuliert. Offensichtlich sind die einzelnen Faktoren der soeben entwickelten Fragestellungen zu eng miteinander verwoben und die Vorgänge zu komplex, als dass eine einzige Perspektive darauf die Antwort liefern könnte. Unter neurologischem Gesichtspunkt geht man weiterhin von Lateralisierung und einer unterschiedlichen Beteiligung der Hirnhälften beim L1und L2-Erwerb aus, jedoch hat sich aufgrund verschiedener Experimente nicht erweisen, „[...] dass es sinnvoll sein könnte, Bedingungen des Zweitsprachenerwerbs weiter mit Hilfe von Lateralitätsexperimenten zu untersuchen“ (LIST: 2001, 697). An verschiedenen Stellen der Fachliteratur wird im Rahmen bestimmter Modelle mit Bezug auf das Zentralnervensystem auf das komplexe Zusammenspiel zwischen zentral-motorischer Programmierung und peripher-motorischen Effektoren hingewiesen; es handelt sich bei Artikulation jeweils um ein „komplexes motorisches Programm für eine bestimmte Bewegung" (RICKHEIT/STROHNER: 2003, 281). Im Zusammenhang mit der Rückmeldung über einen geglückten Bewegungsablauf wird zudem die Bedeutung des Monitors erwähnt. Prozesse des monitoring wiederum spielen in Ansätzen aus der Phonologie eine Rolle, die davon ausgehen, dass die Perzeption bei artikulatorischen Prozessen eine entscheidende Rolle spielt und sich Sprecher auch bei der Sprachproduktion eher an auditiven als an artikulatorischen Eigenschaften der Laute orientieren (HARRIS: 2007, 125).

Mit diesen nur sehr kurz angerissenen und bei weitem nicht vollständigen Verweisen auf die Fachliteratur von Referenzwissenschaften soll deutlich gemacht werden, wie viele unterschiedliche Faktoren bei einer befriedigenden Antwort auf 
die Ausgangsfrage zu berücksichtigen wären. Auf einen Aspekt soll hier jedoch insbesondere zurückgegriffen werden, nämlich die Rolle der Wahrnehmung beim Erlernen der Artikulation.

Dass die Verbindung zwischen Perzeption und Produktion von Lauten ausschlaggebend ist, liegt auf der Hand: Was man nicht hört, kann man auch nur schwer wiedergeben. Nachdrücklich weist Dieling auf diesen Zusammenhang hin (DIELING: 1994, 18 f.).

Liedke stellte darüber hinaus bei einer exemplarischen Lernerbefragung fest, dass die meisten Befragten sich ihres Akzents bewusst waren, ihn jedoch nicht näher bestimmen konnten (LIEDKE: 2007, 398). Falls dies für die Allgemeinheit der Fremdsprachenlerner zutrifft, wird hier erstens festgestellt, dass das unterscheidende Hören Vorsprung gegenüber der artikulatorischen Umsetzung haben kann, und zweitens werden hier klare Argumente für das Bewusstmachen artikulatorischer Vorgänge in Bezug auf die Fremdsprache geliefert, was kognitive Zugänge beim Lernen im Erwachsenenalter nahelegt.

Bongaerts et al. geben einen guten Überblick darüber, welche Bedingungen in der Forschung bis Mitte der Neunziger Jahre als besonders relevant angesehen wurden, um die altersbedingten Unterschiede im Erwerb der Aussprache zu erklären. Dazu gehört die Annahme, dass der Grund in der Wahrnehmung liegt. Genannt werden dabei v. a. Arbeiten von Flege, der sich wiederum auf die Theorie von Wode bezieht. Diese wurde weiter oben bereits in ihren Grundzügen wiedergegeben. Genauer besagt sie, dass es beim Hören von Sprachlauten zwei Wahrnehmungsmodi gibt. Mit dem continuous mode werden jegliche kleinen Unterschiede zwischen Lauten wahrgenommen, der categorical mode, der mit dem Herausbilden eines phonologischen Systems während des Erstspracherwerbs eine steigende Rolle spielt, wird von Kindern während dieses Prozesses zunehmend eingesetzt, um die Signale für bedeutungunterscheidende Laute wahrzunehmen. In der Diskussion um diese Theorie stellte sich die Frage, ob nach Abschluss des Erstspracherwerbs der continuous mode überhaupt nicht mehr aktiviert werden kann oder ob dies lediglich erschwert ist (Bongaerts et al., S. 448 f.). Die Autoren nehmen an, dass der continuous mode von einigen Lernern reaktiviert werden kann, jenen, die die vielzitierten Ausnahmen bilden.

\section{Unter welchen Umständen kann eine native Aussprache erworben werden?}

Ende der Siebziger Jahre führte Gerald Neufeld eine Reihe von Untersuchungen durch, in denen erwachsene Sprecher nach einer Reihe von Trainingsstunden in der Lage waren, Äußerungen in einer Fremdsprache akzentfrei nachzusprechen. Damit wurde gezeigt, dass es sich bei den Einschränkungen in der Aneignung eines neuen phonologischen Systems um keine biologische Notwendigkeit handelt (s. z. B. NEUFELD: 1979). Allerdings waren die Versuchspersonen einem begrenzten 
Vokabular ausgesetzt und mussten sich weder auf semantische noch auf pragmatische Faktoren des Nachgesprochenen konzentrieren. Mit anderen Worten: Es handelte sich um eine reine Imitationsaufgabe, ohne Verständnis dessen, was gesagt wurde. Neufeld selbst stellt die Frage, inwieweit das erfolgreiche Nachsprechen isolierter Äußerungen auf reale Kommunikationssituationen mit der Notwendigkeit, selbst Äußerungen generieren zu müssen, übertragbar ist (ebd., S. 234). Bei weiteren Versuchen, in denen Sprecher mit nahezu muttersprachlichem Akzent Texte in der Zweitsprache vorlesen mussten und zum Teil von Testpersonen für Muttersprachler gehalten wurden, beschränkt sich bereits in der Anordnung dieses Versuchs das Feld auf eine bestimmte Auswahl von Sprechern (ebd., S. 235). In der Tat bemerkt Bose: „In dem Augenblick, in dem die Deutschlernenden wirklich kommunizieren, [...] achten sie nicht mehr auf die mühsam antrainierten phonetischen Merkmale. Falls diese nicht wirklich automatisiert sind, wird auf die muttersprachliche Artikulationsbasis auch in der Fremdsprache zurückgegriffen" (BOSE: 1994, 131).

Bongaerts et al. knüpfen in ihren zwei Versuchsreihen an Neufeld an, indem sie ihrerseits nachzuweisen versuchen, dass der Erwerb einer authentischen Aussprache im Erwachsenenalter möglich ist. Dabei gehen sie auf die zweifelsfreien Errungenschaften seines Versuchs zurück, indem sie annehmen, dass die Fertigkeiten in Wahrnehmung und Motorik, die für eine akzentfreie Artikulation nötig sind, auch bei Erwachsenen vorhanden bzw. aktivierbar sind (BONGAERTS et al.: 1997, 450). In den Versuchsanordnungen ist jedoch vor allem das Ziel, die Schwächen jener frühen Experimente zu vermeiden. Die Versuchspersonen mit niederländischer L1 sind sehr fortgeschrittene Lerner des Englischen, die jedoch erst in den Studienjahren an der Universität intensiven Input durch Muttersprachler erhalten haben. Es handelte sich nicht um Imitationsübungen, sondern um das genuine Produzieren von Äußerungen, die von den Sprechern selbst verstanden wurden. Diese bestanden zum Teil aus freiem Sprechen, zum Teil aus dem Vorlesen ganzer Sätze mit jeweils konzentrierten phonologischen Fallstricken. Im Zusammenhang mit der Wichtigkeit des Reaktivierens des continuous mode weisen Bongaerts et al. darauf hin, dass es bei einem etablierten phonologischen System in der L1 für den Lerner vor allem schwierig ist, Unterschiede zwischen solchen Lauten wahrzunehmen, die sich in der L1 und der L2 phonetisch sehr ähnlich sind (ebd., S. 449). Ein Beispiel wären Plosiva in den germanischen Sprachen, die unterschiedliche voice onset time haben können.

Um die Ergebnisse dieser Versuche kurz zusammenzufassen: Es konnte nachgewiesen werden, dass die fünf von elfVertretern dieser bestimmten Lernergruppe von den Mutter-sprachlern, die die Äußerungen beurteilten, statistisch signifikant als muttersprachlich einge-stuft wurden. Dieser Beurteilung hielten sie auch bei einer Auswertung der Ergebnisse stand, die mögliche Schwächen bei einzelnen Sätzen berücksichtigte, mit denen sie ihre Identität als Nichtmuttersprachler eventuell hätten preisgeben können. 
Die Autoren dieser Studie sehen ihre Annahme, dass der Erwerb einer authentischen Aussprache im Erwachsenenalter möglich ist, also bestätigt, stellen jedoch fest, dass diese Lerner Ausnahmen darstellen. Insofern ergibt sich hier kein Unterschied zu den üblichen Aussagen zu dieser Frage, es wird jedoch spezifiziert, welche Bedingungen bei dieser Sprechergruppe vorgelegen haben. Folgende Aspekte werden dabei als wahrscheinliche Einflussfaktoren genannt: größere neurokognitive Flexibilität, die bewirkt, dass Einschränkungen durch eine (angenommene) kritische Periode nicht gelten, außergewöhnliche Hirnstrukturen in Bezug auf Sprache, sehr hohe Motivation (die Versuchspersonen unterrichteten alle Englisch an der Universität) sowie gezielter sprachlicher Input über Wahrnehmungstraining (ebd., S. 462 f.). Das Autorenkollektiv geht davon aus, dass ein solches Training in der Unterrichtspraxis einem weiteren vorauszugehen habe, in dem die Feinabstimmung auch in der Produktion von Sprachlauten erreicht werden könne (ebd., S. 463).

\section{Welche Variablen beeinflussen das Erlernen der Aussprache?}

Mit der Nennung einiger Merkmale dieser „,highly successful learners“ (ebd., S. 447) ergibt sich der Übergang zu einem weiteren Fragekomplex, nämlich, welche Faktoren beim Erlernen der Aussprache im Erwachsenenalter besonders berücksichtigt werden müssen und wie dies im Unterricht umzusetzen ist. Zusätzlich zu den eben genannten finden sich in der Fachliteratur zahlreiche Variable, die sich jedoch zumeist auf den L2-Erwerb im Allgemeinen beziehen. Sie lassen sich in sprachliche, individuelle und soziale Variable unterscheiden (OKSAAR: 2003, 61). Ohne auf jede einzelne dieser Faktoren näher eingehen zu wollen, sei hier eine Reihe von Einflüssen genannt, die insbesondere für die Aussprache zum Tragen kommen können. Selbstverständlich ist die Bedeutung der einzelnen Wirkungsmomente stark individuell und abhängig von der jeweiligen Lernerpersönlichkeit. Dies schließt jedoch nicht aus, dass sie jeweils für sich auf größere Lernergruppen zutreffen und berücksichtigt werden müssen.

Zunächst einmal gibt es die Möglichkeit, dass jemand aus irgendeinem Grund lautliche Unterschiede besser „hört“, sei es aus Gründen der bisherigen Kontakte mit Fremdsprachen, also einer gewissen Sensibilisierung und Übung, sei es aus Gründen der sogenannten Sprachlerneignung oder vielleicht aus Gründen besonderer Strukturen, wie sie in der Hirnforschung untersucht werden. Auch hier gibt es noch einige offene Fragen. Besteht zum Beispiel einen Zusammenhang zwischen genauem musikalischen Hören und der Fähigkeit, den continuous mode eher zu aktivieren, d. h. funktioniert eine übergeordnete Fähigkeit zum fine tuning?

Um bei den phonologischen Faktoren zu bleiben, sei noch einmal der theoretische Hintergrund zu den Versuchen von Bongaerts et al. erwähnt: Wenn das phonologische System in der L1 etabliert ist, dann ist für den Lerner vor allem schwer, Unterschiede zwischen solchen Lauten wahrzunehmen, die sich in der L1 und der L2 phonetisch sehr ähnlich sind. Daraus lassen sich Schlussfolgerungen für Lehr- und Lernmethoden ziehen. 
Viele Lehrbücher legen bei phonetischen Übungen den Schwerpunkt auf Laute, die für die L2 spezifisch sind. Einige bekannte Phänomene, wie das Nichtunterscheidenkönnen von [r] und [l] etwa durch japanische Sprecher, werden zusätzlich einbezogen. Im Allgemeinen fallen jedoch solche Laute durch das Raster, die es (angeblich) in beiden Sprachen gibt, z. B. eine Reihe von Plosiva. An Taschenwörterbüchern etwa für den Reisebedarf ist das am deutlichsten zu sehen, wenn etwa eine beliebte Anmerkung zu einem bestimmten Buchstaben lautet „wie im Deutschen“1. Gerade da kann aber der Teufel wörtlich im Detail stecken und minimale Unterschiede in der jeweiligen Artikulation unentdeckt und damit auch unrealisiert bleiben. Es ist wünschenswert, dass die marktgängigen Lehrund Übungsbücher mehr Wert auf die angeblich unproblematischen Laute legen, zumindest wenn sie sich an eine bestimmte Zielgruppe richten. An Publikationen wie Reisesprachführer denselben Maßstab anzulegen, wäre selbstverständlich zweckund realitätsfremd: Ein Reisewörterbuch kann keine phonologische Abhandlung sein, und für jemand, der sich für einen nur kurzen Zeitraum notdürftig verständigen will, ist das Ziel sicher nicht eine authentische Aussprache oder gar Integration in die Gastkultur.

Bei den individuellen und sozialen Variablen seien zunächst einige allgemeine Voraussetzungen genannt. Oksaar betont die zentrale Rolle der Imitation nicht nur beim Erst- sondern auch beim Zweitspracherwerb. Es sei in der Forschung bisher nicht widerlegt, dass Kinder eine bessere Imitationsfähigkeit besitzen als Erwachsene (OKSAAR: 2003, 57). Allerdings weist sie auf den vernachlässigten Aspekt hin, „dass spontane Imitationen auch verschiedene kommunikative, z. B. phatische Funktionen haben können, oder emphatische Übereinstimmung mit dem Gesprächspartner ausdrücken" (ebd., S. 57 f.). Daraus ergeben sich direkte Konsequenzen für die Unterrichtssituation. Da die kommunikative Rolle der Imitation bisher jedoch in der Forschung kaum eine Rolle gespielt hat, ist anzunehmen, dass auch das Entwickeln entsprechender Strategien und Methoden für den Unterricht auf sich warten lassen wird.

In diesem Zusammenhang erwähnt Oksaar auch die Flexibilität und Spontaneität von Vorschulkindern, mit der diese an Sprache herangehen. Daraus wird zunächst der Schluss gezogen, dass ein früher Zweitsprachenbeginn wünschenswert ist, für die Zwecke der hier vorgelegten Problemstellung muss jedoch gefragt werden, wie in einer Lernsituation bei Erwachsenen Hemmungen abgebaut und Spontaneität gefördert werden kann. Dies könnte vor allem in Verbindung damit nützlich sein, Wege zu finden, wie man erwachsenen Lernern den Zugang zu dem oben erwähnten continuous mode wieder ermöglichen kann. Das aktive Abweichen vom erworbenen phonologischen System dürfte nicht nur eine Frage des gezielten Perzeptions- und

1 Ein völlig willkürlich herausgegriffenes Beispiel findet sich in Langenscheidts Sprachführer Tschechisch, wo die Erläuterung für das tschechische Graphem $<\mathrm{h}>$ lautet „wie h in Hund", ungeachtet des Vorhandenseins oder Fehlens von Stimmhaftigkeit (S. 13). 
Produktionstrainings sein, sondern gerade in einer Unterrichtssituation werden soziale und affektive Gegebenheiten eine Rolle spielen, die solches Abweichen erschweren oder fördern. Auf psychosoziale Faktoren soll gleich noch Bezug genommen werden.

Westhoff nennt unter „affektive und soziale Strategien“ einige Übungen, die der Lockerung und Entspannung dienen sollen, räumt aber ein, dass sich die Effektivität solcher Aktivitäten empirisch bisher wenig beweisen lässt (WESTHOFF: 2001, 687 f.). Im Einzelnen sind dies „Atemübungen, entspannende Musik, entspannendes Gelächter, rhythmische Wiederholungen von gewissen Bewegungen oder einsilbigen Wörtern“ (ebd., S. 687). Meiner Ansicht nach nutzen solche Übungen wenig, wenn sie in einer gespannten oder formalen Atmosphäre stattfinden. Wahrscheinlich ist effektiver, vor allem für eine lockere, heitere Unterrichtsumgebung zu sorgen, in der Humor, Spielen mit Identitäten, Rollen und der Stimme sowie freies Sichbewegen ihren Platz haben. Dies lässt sich weniger organisieren und formalisieren als vorgegebene Übungen etwa zum entspannenden Gelächter, kann aber letztlich den erforderlichen Raum für das Ausprobieren von neuen Verhaltensweisen schaffen auch artikulatorisch.

Das Spielen mit Rollen und Identitäten könnte tatsächlich ein Schlüssel zur positiven Gestaltung von Lernervariablen sein. Denn als entscheidende übergeordnete Kriterien einflussreicher Variablen benennt Oksaar Motivation und Attitüden. Von Motivation war bereits im Zusammenhang mit den Versuchspersonen bei Bongaerts et al. die Rede. Zielgerichtetheit und der feste Wunsch, das Ziel zu erreichen (selbst wenn die Gründe extrinsisch sind) scheinen maßgebliche Faktoren zu sein. Die Rolle der Attitüden ist vor allem bei Migranten untersucht worden und steht dort in Wechselwirkung mit der gesamten Lebenssituation. Zufriedenheit mit der Situation und der Wunsch nach bzw. das Erreichen von Integration können sich positiv auswirken (OKSAAR: 2003, 64). Oksaar geht sogar so weit anzunehmen, dass - da neurophysiologische Erklärungen für das Vorhandensein eines Akzents im Erwachsenenalter nicht ausreichen - in Wirklichkeit eine innere Hemmung vorliegt: „es handelt sich nicht um ein Nicht-Können, sondern eher um ein bewusstes oder unbewusstes Nicht-Wollen" (ebd.). Die persönliche und soziale Identität, die auch durch Sprache geformt wird, würde durch das akzentfreie Verwenden der Fremdsprache gestört, der Akzent sei ein „Identitätsmarkör“" (ebd.). Sie belegt das durch Studien, in denen Erwachsene angeben, sich in solchen Situationen wie ein Schauspieler zu fühlen. Der wechselweise Karl V., Goethe und Schopenhauer nachgesagte Ausspruch „So viele Sprachen einer kann, so viele Male ist er Mensch“ ist offenbar keine Konstellation, in der sich jeder Lerner ohne weiteres wohlfühlt.

Dieses Hindernis könnte m. E. aber auch positiv genutzt werden. An anderer Stelle berichtet Oksaar nämlich von zwei Männern, die innerhalb weniger Wochen vom Sprechen mit Akzent zu akzentfreiem Englisch gekommen seien, um eine Filmrolle zu erhalten. Wenn die Fremdsprache tatsächlich nur akzentfrei 
gesprochen werden kann, wenn man in eine fremde Rolle schlüpft, sollten Situationen geschaffen werden, in denen dies möglich ist, ohne die eigene Identität aufgeben zu müssen. Der oben angedeutete Raum für das Spielen mit Rollen und Identitäten in Verbindung mit der Ausspracheschulung ist eine Aufgabe für die Fremdsprachendidaktik. Um es kühn zu formulieren: Möglicherweise wären die in verschiedenen wissenschaftlichen Artikeln hervorgehobenen Ausnahmefälle keine Ausnahmen, wenn der Fremdsprachenunterricht für Erwachsene neue Wege gehen würde.

Mit diesen einflussreichen Variablen und Methoden konnten nur einige aus einer Fülle von Einflüssen genannt werden, die auf den erwachsenen Lerner einwirken bzw. die man sich im Unterricht zunutze machen kann. Die Fachliteratur zum Fremdspracherwerb bietet inzwischen wesentlich mehr Vorschläge, von denen im Hinblick auf Sprachlautperzeption und -rezeption vor allem diejenigen förderlich sind, die die ganze Lernerpersönlichkeit zu einer Erweiterung des eigenen Wahrnehmungs- und Verhaltenshorizonts anregen.

\section{Zusammenfassung und Ausblick}

In diesem Beitrag sollte darauf aufmerksam gemacht werden, dass trotz der nicht anzuzweifelnden Erfahrung, dass Lerner im Erwachsenenalter Schwierigkeiten haben, eine native Aussprache in der L2 zu ereichen, diesbezüglich einige Fragen offen sind. Sie sollen darauf abzielen, neueste Forschungsergebnisse aus den Nachbardisziplinen in Theorien zum Fremdspracherwerb zur Kenntnis zu nehmen und Konsequenzen für die Unterrichtspraxis zu ziehen. Zu fragen ist insbesondere, unter welchen Umständen erwachsene Lerner in der Lage sind, eine authentische Aussprache in der L2 zu erwerben und welche Faktoren eine stärkere Rolle spielen als andere. Eine geeignete Methoden- und Übungstypologie, die die verschiedenen Variablen in spezifischer Weise berücksichtigt, wäre die Konsequenz für die Anwendung im Unterricht. Insofern ergeben sich aus den Fragen in diesem Beitrag auch Forderungen an die Wissenschaft, sowohl an linguistische Disziplinen als auch an die Referenzwissenschaften, vor allem aber dahingehend, sich gegenseitig stärker wahrzunehmen.

\section{Literatur:}

APELTAUER (2001): Apeltauer, Ernst. Zweitsprachenerwerb als Lernaktivität I: Lernersprache - Lernprozesse - Lernprobleme, in: Helbig, Gerhard/Götze, Lutz/Henrici, Gert/Krumm, Hans-Jürgen: Deutsch als Fremdsprache. Ein internationales Handbuch. 1. Halbband (= Handbücher zur Sprach- und Kommunikationswissenschaft, Band 19.1). Berlin/New York: de Gruyter, 2001, 677-684.

BIRDSONG (1999): Birdsong, David (Hg.). Second language acquisition and the critical period hypothesis. Mahwah: Lawrence Erlbaum Associates, 1999. 
BONGAERTS (1999): Bongaerts, Theo. Ultimate attainment in 12 pronunciation: the case of very advanced late L2 learners, in: Birdsong, David (Hg.): Second language acquisition and the critical period hypothesis. Mahwah: Lawrence Erlbaum Associates, 1999, 133-160.

BONGAERTS et al. (1997): Bongaerts, Theo/van Summeren, Chantal/Planken, Brigitte/ Schils, Erik: Age and ultimate attainment in the pronunciation of a foreign language, in: Studies in Second Language Acquisition, 1997, 19, Nr. 4, 447-465.

BOSE (1994): Bose, Ines. Gesprächsfähigkeit als Ziel im Fremdsprachenunterricht Deutsch für Fortgeschrittene - Ansätze zur Verbindung von Arbeitsmethoden aus Phonetik und Rhetorik, in Breitung, Horst (Hg.): Phonetik. Intonation. Kommunikation. (= Standpunkte zur Sprach- und Kulturvermittlung 2. Werkstattberichte des Goethe-Instituts). München: Goethe-Institut 1994, 129-140.

BUTZKAMM (2002): Butzkamm, Wolfgang. Psycholinguistik des Fremdsprachenunterrichts. Von der Muttersprache zur Fremdsprache. Tübingen/Basel: Francke, 3., neubearb. Aufl., 2002.

DIELING (1994): Dieling, Helga. Phonetik in Lehrwerken Deutsch als Fremdsprache von 1980 bis 1992. Eine Analyse, in: Breitung, Horst (Hg.): Phonetik. Intonation. Kommunikation. (= Standpunkte zur Sprach- und Kulturvermittlung 2. Werkstattberichte des Goethe-Instituts). München: Goethe-Institut 1994, 13-20.

FIEHLER (2003): Fiehler, Reinhard. Spracherwerb im Erwachsenenalter, in: Rickheit, Gerd/Herrmann, Theo/Deutsch, Werner (Hg.): Psycholinguistik / Psycholinguistics. Ein internationales Handbuch (= Handbücher zur Sprach- und Kommunikationswissenschaft, Band 24). Berlin/New York: de Gruyter, 2003, 812-819.

GASS/SELINKER (2001): Gass, Susan M./Selinker, Larry. Second language acquisition. An introductory course. Mahwah, London: Lawrence Erlbaum Associates, 2001.

HARRIS (2007): Harris, John. Representation, in: de Lacy, Paul (Hg.): The Cambridge handbook of phonology. Cambridge u.a.: Cambridge University Press, 2007, 119-137.

JORDENS (2003): Jordens, Peter. Constraints on the shape of second language learner varieties, in: Rickheit, Gerd/Herrmann, Theo/Deutsch, Werner (Hg.): Psycholinguistik / Psycholinguistics. Ein internationales Handbuch (= Handbücher zur Sprach- und Kommunikationswissenschaft, Band 24). Berlin/New York: de Gruyter, 2003, 819-833.

KLEIN (2008): Klein, Wolfgang: Die Werke der Sprache. Für ein neues Verhältnis zwischen Literaturwissenschaft und Linguistik, in: Zeitschrift für Literaturwissenschaft und Linguistik, 2008, Nr. 150, 8-32.

Langenscheidts Sprachführer Tschechisch mit Reisewörterbuch (1996). Berlin, München: Langenscheidt, 1996.

LENNEBERG (1967): Lenneberg, Eric H. Biological foundations of language. New York: John Wiley, 1967.

LIEDKE (2007): Liedke, Martina. Deutsch als Fremdsprache, in: Knapp, Karlfried et al. (Hg.): Angewandte Linguistik. Ein Lehrbuch. Tübingen und Basel: A. Francke Verlag, 2., überarb. u. erw. Aufl., 2007, 389-410.

LIST (2001): List, Gudula. Zweitsprachenerwerb als individueller Prozess I: Neuropsychologische Ansätze, in: Helbig, Gerhard/Götze, Lutz/Henrici, Gert/Krumm, Hans-Jürgen: Deutsch als Fremdsprache. Ein internationales Handbuch. 1. Halbband (= Handbücher zur Sprach- und Kommunikationswissenschaft, Band 19.1). Berlin/New York: de Gruyter, 2001, 693-700.

NEUFELD (1979): Neufeld, Gerald G. Towards a theory of language learning ability, in: Language Learning. A Journal of Applied Linguistics, 1979, 29, Nr. 2, 227-241.

OKSAAR (2003): Oksaar, Els. Wege zur Mehrsprachigkeitund zur interkulturellen Verständigung. Stuttgart: Kohlhammer, 2003. 
RICKHEIT/STROHNER (2003): Modelle der Sprachproduktion, in: Herrmann, Theo/Grabowski, Joachim (Hg.): Sprachproduktion (= Enzyklopädie der Psychologie, Thermenbereich C: Theorie und Forschung, Serie III: Sprache, Band 1). Göttingen/Bern/ Toronto/Seattle: Hogrefe, 2003, 267-286.

SCHÖNPFLUG (2001): Schönpflug, Ute. Zweitspracherwerb als individueller Prozess II:

Biologische und neurophysiologische Grundlagen, in: Helbig, Gerhard/Götze, Lutz/ Henrici, Gert, Krumm/Hans-Jürgen: Deutsch als Fremdsprache. Ein internationales Handbuch. 1. Halbband (= Handbücher zur Sprach- und Kommunikationswissenschaft, Band 19.1). Berlin/New York: de Gruyter, 2001, 701-706.

WESTHOFF (2001): Westhoff, Gerard J. Zweitsprachenerwerb als Lernaktivität II: Lernstrategien - Kommunikationsstrategien - Lerntechniken, in: Helbig, Gerhard/Götze, Lutz/Henrici, Gert/Krumm, Hans-Jürgen: Deutsch als Fremdsprache. Ein internationales Handbuch. 1. Halbband (= Handbücher zur Sprach- und Kommunikationswissenschaft, Band 19.1). Berlin/New York: de Gruyter, 2001, 684-692.

WODE (1996): Wode, Henning. Speech perception and L2 phonological acquisition, in: Jordens, Peter/Lallemann, Josine (Hg.): Investigating second language acquisition. Berlin, New York: de Gruyter, 1996, 321-353. 